\title{
On the rare species biology of the genus Iris $L$. in culture and natural habitats in the South Urals
}

\author{
Anastasia V. Kryukova ${ }^{1}$, Alfiya N. Mustafina ${ }^{1}$, and Larisa Abramova ${ }^{1 *}$ \\ ${ }^{1}$ South Ural Botanical Garden Institute of Ufa Federal Research Center of the Russian Academy of \\ Sciences, 450080, Ufa, Russia
}

\begin{abstract}
The article represents a comparison of rare species morphometric indicators and seed productivity of the genus Iris in the South Urals. It's shown that I. scariosa, I. humilis and I. pseudacorus habitus deteriorate and seed productivity decreases under culture conditions, but I. pumila samples in culture outstrip plants in natural habitats. I. pumila is promising for cultivation in the South Ural region
\end{abstract}

\section{Introduction}

Species of the genus Iris L. are characterized with high decorative qualities; since the ancient times they have been introduced into flower culture and widely used in settlement greening, erven, gardens. There are a number of wild species promising for cultivation and breeding found in the natural flora. Many wild species of the genus are included in the Red Books of various regions, because they disappear under the influence of anthropogenic factors. Studying their biology, reproduction and other characteristics has not only important biological significance, but allows us to recommend the most resistant species with valuable decorative qualities to expand the range of flower-decorative plants.

\section{Materials and methods}

The research area is the Republic of Bashkortostan and adjacent territories in Orenburg and Chelyabinsk Regions. Field studies were carried out in 2012-2017 at 23 natural coenopopulations (CPs) of rare decorative species of the genus Iris - Iris pumila L. (11 CPs), I. scariosa Willd. ex Link (5 CPs), I. humilis Georgi (1 CP) and I. pseudacorus L. (6 CPs). All studied species are listed in the Red Book of the Republic of Bashkortostan (RB) [1] (2011) and 2 species (Iris pumila, I. scariosa) are included in the Red Book of the Russian Federation (RF) [2] (2008).

To test for introduction, Iris species planting material was mobilized by small rhizome fragments with 1-2 shoots (10-30 units each): I. pumila - in 2009 from 7 CPs of Pre-Urals, I. pseudacorus - in 1987 and 2012 from Ufa Region, I. scariosa - in 2013 from

\footnotetext{
* Corresponding author: abramova.lm@mail.ru
} 
Khaibullinsky district, I. humilis - in 2014 from the Uchalinsky district (both in TransUrals, RB).

The introduction test was done in the South Ural Botanical Garden Institute of UFRC RAS (Ufa). The introducing conditions corresponded to a greater extent to the natural conditions for Iris pumila habitats, but significantly varied for the other three species, two of which grow in Trans-Urals, and I. pseudacorus - in quite different environmental conditions (wet meadows).

Studying morphometry in culture and natural conditions was carried out according to V.N. Golubev [3] approach in the flowering phase on 25 average-generating individuals of each CP; a counting unit was one annual shoot. The following parameters were taken into account: clone diameter $(\mathrm{dc}), \mathrm{cm}$; number of vegetative escapes $(\mathrm{Ne})$, pcs.; number of generative shoots per 1 plant (Ngs), pcs.; generative shoot height $(\mathrm{h}), \mathrm{cm}$; length $(\mathrm{Ll})$ and width of a generative shoot second leaf (Sl), cm; length (Llpp) and width of the lower perianth lobe (Slpp), cm; length (Lupp) and width of the upper perianth lobe (Supp), cm; flower diameter (dfl), $\mathrm{cm}$.

Seed productivity was determined by a standard technique [4] (Vainagiy, 1974). The number of flowers $(\mathrm{Nfl})$ and fruits $(\mathrm{Nfr})$ per a generative shoot was accounted; real (RSP) and potential (PSP) seed productivity, and seed productivity coefficient (Isp, \%) were estimated.

Statistical analysis was realized in MS Excel 2010 applying the statistical software package Statistica 10 [5] (Zaitsev, 1990). To compare the mean values, Student's t-test was used (at 5\% significance level) [6] (Khalafyan, 2008).

\section{Results}

The species morphometric indicators comparison of the Iris genus in natural habitats and introduction according to the averaged data of all CPs of each species for 4-6 years of research (Table 1) has shown that reliable differences between plants at a 5\% significance level are revealed for most parameters of I. pumila and I. pseudacorus, but the differences are mostly unreliable (at $\mathrm{t}_{\text {fact }}<2.064$ ) for other two species.

I. scariosa, I. humilis, and I. pseudacorus habitus is worthening under culture conditions. I. pumila in culture surpasses plants in natural habitats. The differences are caused, first of all, by plant treatment in culture, higher soil moisture compared to the species natural habitats, and the lack of competition with other plant species.

Table 1. Comparison of long-term morphometric parameters of four species of the genus Iris in nature and culture

\begin{tabular}{|c|c|c|c|c|}
\hline \multirow{2}{*}{ Parameters } & I. pumila & I. scariosa & I. humilis & I. pseudacorus \\
\cline { 2 - 5 } & \multicolumn{4}{|c|}{ Environment/Culture } \\
\hline \multirow{2}{*}{$\mathrm{dc}$} & $\underline{19.6 \pm 1.15}$ & $\underline{43.0 \pm 1.42}$ & $\underline{16.0 \pm 1.89}$ & $\underline{\underline{76.6 \pm 4.08}}$ \\
\hline \multirow{2}{*}{$\mathrm{Ne}$} & $\underline{25.9 \pm 2.46}$ & $13.0 \pm 0.63$ & $11.2 \pm 0.31$ & $27.2 \pm 1.17$ \\
\hline \multirow{2}{*}{$\mathrm{Ll}$} & $55.1 \pm 4.52$ & $\underline{62.7 \pm 3.20}$ & $\underline{7.8 \pm 1.06}$ & $\underline{34.3 \pm 1.47}$ \\
& $\underline{14.6 \pm 1.03}$ & $\underline{13.7 \pm 0.27}$ & $\underline{10.0 \pm 0.36}$ & $6.8 \pm 0.41$ \\
\hline
\end{tabular}




\begin{tabular}{|c|c|c|c|c|}
\hline $\mathrm{S} 1$ & $\frac{1.3 \pm 0.14}{1.8 \pm 0.15}$ & $\frac{1.4 \pm 0.02}{1.3 \pm 0.04}$ & $\frac{0.9 \pm 0.05}{1.2 \pm 0.05}$ & $\frac{2.4 \pm 0.04}{1.5 \pm 0.08}$ \\
\hline Ngs & $\frac{5.0 \pm 0.35}{22.8 \pm 2.18}$ & $\frac{16.6 \pm 0.81}{2.4 \pm 0.27}$ & $\frac{3.5 \pm 0.26}{1.7 \pm 0.26}$ & $\frac{6.5 \pm 0.31}{1.5 \pm 0.14}$ \\
\hline $\mathrm{h}$ & $\frac{16.1 \pm 0.50}{17.2 \pm 1.44}$ & $\frac{16.1 \pm 0.29}{12.5 \pm 0.31}$ & $\frac{8.2 \pm 0.23}{8.7 \pm 0.21}$ & $\frac{87.6 \pm 2.29}{42.7 \pm 3.16}$ \\
\hline Llpp & $\frac{4.9 \pm 0.05}{7.8 \pm 0.21}$ & $\frac{5.3 \pm 0.07}{5.1 \pm 0.11}$ & $\frac{4.7 \pm 0.18}{5.9 \pm 0.21}$ & $\frac{5.9 \pm 0.07}{5.2 \pm 0.11}$ \\
\hline Slpp & $\frac{1.6 \pm 0.03}{3.7 \pm 0.12}$ & $\frac{1.7 \pm 0.02}{1.3 \pm 0.03}$ & $\frac{1.0 \pm 0.02}{4.4 \pm 0.05}$ & $\frac{3.1 \pm 0.03}{3.4 \pm 0.06}$ \\
\hline Lupp & $\frac{4.8 \pm 0.05}{4.8 \pm 0.41}$ & $\frac{4.7 \pm 0.08}{4.4 \pm 0.14}$ & $\frac{2.7 \pm 0.04}{3.2 \pm 0.15}$ & $\frac{2.5 \pm 0.04}{2.3 \pm 0.04}$ \\
\hline Supp & $\frac{1.5 \pm 0.02}{2.3 \pm 0.11}$ & $\frac{1.6 \pm 0.02}{1.3 \pm 0.03}$ & $\frac{0.9 \pm 0.02}{1.3 \pm 0.04}$ & $\frac{0.6 \pm 0.02}{0.9 \pm 0.02}$ \\
\hline $\mathrm{dfl}$ & $\frac{5.3 \pm 0.10}{6.7 \pm 0.38}$ & $\frac{6.2 \pm 0.09}{5.4 \pm 0.12}$ & $\frac{4.9 \pm 0.04}{5.4 \pm 0.06}$ & $\frac{8.8 \pm 0.13}{7.8 \pm 0.13}$ \\
\hline
\end{tabular}

Note: unreliable differences by the Student criterion are italicized, the remaining indicators are reliable at $5 \%$ significance level.

Investigations of the long-term average seed productivity of Iris species (Table 2) has demonstrated that I. scariosa, I. humilis, and I. pseudacorus have average values of the most reproductive parameters under conditions of introduction significantly lower than the average values obtained in natural populations.

Table 2. Comparison of seed productivity elements of Iris rare species in culture and environment

\begin{tabular}{|c|c|c|c|c|}
\hline \multirow{2}{*}{ Parameters } & I. pumila & I. scariosa & I. humilis & I. pseudacorus \\
\cline { 2 - 5 } & \multicolumn{4}{|c|}{ Environment/Culture } \\
\hline \multirow{2}{*}{ Nfl } & $\underline{1.0 \pm 0.00}$ & $\underline{1.9 \pm 0,16}$ & $\underline{1.7 \pm 0.05}$ & $\underline{11.5 \pm 0.45}$ \\
\hline \multirow{2}{*}{ Nfr } & $1.0 \pm 0.00$ & $1.6 \pm 0,13$ & $1.4 \pm 0.05$ & $8.4 \pm 0.35$ \\
\hline \multirow{2}{*}{ RSP } & $\underline{1.0 \pm 0.00}$ & $\underline{1.7 \pm 0,30}$ & $\underline{1.2 \pm 0.15}$ & $\underline{7.9 \pm 0.22}$ \\
\hline \multirow{2}{*}{ PSP } & $\underline{1.0 \pm 0.00}$ & $1.2 \pm 0,20$ & $1.0 \pm 0.15$ & $5.9 \pm 0.48$ \\
\hline & $\underline{29.4 \pm 0.52}$ & $\underline{45.9 \pm 1,68}$ & $\underline{11.6 \pm 0.46}$ & $\underline{561.8 \pm 78.3}$ \\
& $52.7 \pm 1.47$ & $12.5 \pm 0,55$ & $5.8 \pm 0.42$ & $317.1 \pm 48.5$ \\
\hline
\end{tabular}




\begin{tabular}{|c|l|l|l|l|}
\hline $\mathrm{I}_{\mathrm{sp}}$ & $\underline{59.5 \pm 1.52}$ & $\underline{61.2 \pm 1,44}$ & $\underline{43.3 \pm 1.35}$ & $\underline{81.6 \pm 1.51}$ \\
\hline & $68.7 \pm 1.39$ & $55.0 \pm 1,56$ & $56.8 \pm 1.23$ & $72.0 \pm 1.23$ \\
\hline
\end{tabular}

Note: unreliable differences by Student criterion are italicized

Assessment of I. pumila seed productivity has shown the most reproductive parameters reliably exceed similar parameters under natural then cultural conditions, that is a significant indicator of a species adaptation in culture.

I. pumila is the most promising for cultivation in the South Ural region.

\section{References}

1. The Red Book of the Republic of Bashkortostan. Vol. 1: Plants and fungi (MediaPrint, Ufa, 2011)

2. The Russian Federation Red Book (plants and fungi) (KMK, Moscow, 2008)

3. V.N. Golubev, Proceedings of V.V. Alekhin Central Black Earth Reserve, 7 (1962)

4. I.V. Vainagy, Bot. J. 59, 826-831 (1974)

5. G.N. Zaitsev, Maths in experimental botany (Nauka, Moscow, 1990)

6. A.A. Khalafyan, Statistica 6. Data statistical analysis (Binom, Moscow, 2008) 